\title{
L'ATTRACTIVITÉ SANS LE TERRITOIRE ? L'INDUSTRIE MAROCAINE A-T-ELLE BESOIN DU TERRITOIRE ?
}

Jean-Luc Piermay

Armand Colin | «Annales de géographie »

$2011 / 2 \mathrm{n}^{\circ} 678$ | pages 204 à 215

ISSN 0003-4010

ISBN 9782200926946

Article disponible en ligne à l'adresse :

http://www.cairn.info/revue-annales-de-geographie-2011-2-page-204.htm

\section{Pour citer cet article :}

Jean-Luc Piermay, «L'attractivité sans le territoire ? L'industrie marocaine a-t-elle besoin du territoire ? », Annales de géographie 2011/2 (n678), p. 204-215.

DOI 10.3917/ag.678.0204

Distribution électronique Cairn.info pour Armand Colin.

(C) Armand Colin. Tous droits réservés pour tous pays.

La reproduction ou représentation de cet article, notamment par photocopie, n'est autorisée que dans les limites des conditions générales d'utilisation du site ou, le cas échéant, des conditions générales de la licence souscrite par votre établissement. Toute autre reproduction ou représentation, en tout ou partie, sous quelque forme et de quelque manière que ce soit, est interdite sauf accord préalable et écrit de l'éditeur, en dehors des cas prévus par la législation en vigueur en France. Il est précisé que son stockage dans une base de données est également interdit. 


\title{
L'attractivité sans le territoire ? L'industrie marocaine a-t-elle besoin du territoire?
}

\author{
Jean-Luc Piermay \\ Professeur de géographie, Université de Strasbourg, Laboratoire Systèmes géographiques \\ complexes
}

Cet article étudie la manière dont s'articulent l'entreprise industrielle et l'espace local, dans le cadre d'une économie en changement, celle du Maroc, dont une particularité forte est que l'ambition du développement vient du sommet de l'État et que le milieu local n'y est pas associé a priori. Il s'agit donc d'observer si, puis comment, une territorialisation se fait malgré tout localement autour de la question productive. Au-delà de l'encastrement reconnu de l'économie dans les dynamiques sociales, il s'agit ici de replacer le productif dans une perspective plus large, afin de mieux effectuer le bouclage entre le fonctionnement de l'entreprise et celui de l'espace (Rallet et Torre, 2007 ; Burger et al., 2009). L'hypothèse est que, pour comprendre l'interaction entre l'entreprise et l'espace, entre les performances économiques des entreprises et les faits d'agglomération, il est nécessaire de déporter le regard hors de la sphère productive, en observant l'ensemble des processus de territorialisation, y compris non liés à la production, qui se nouent autour de l'entreprise. Cette hypothèse est en phase avec les remarques méthodologiques émises pour les économies du Sud par des auteurs d'un ouvrage collectif (Antheaume et al., 2005). Pecqueur propose d'intégrer réellement les relations hors marché à l'analyse; Fauré et al. conseillent de considérer avec prudence les configurations productives locales, susceptibles de contenir de la "modernité régressive », et appellent pour cela à interroger les soubassements anthropologiques et culturels des territoires de la production.

\section{Un intérêt modeste pour le territoire}

Il ne s'agit pas ici de retracer la politique industrielle marocaine (Piermay et al., 2009), mais de montrer comment elle prend en compte les idées de proximité et de territoire. On pourrait dire que si la proximité occupe dans l'industrie marocaine une place importante, le territoire n'a dans les politiques publiques qu'une place modeste.

La proximité, c'est la forte concentration des activités dans la métropole économique, Casablanca. Avec 3500000 habitants, un peu plus de $10 \%$ de la population du pays, la Région du Grand Casablanca regroupait 54,9\% de l'emploi industriel en 1990 et $44 \%$ en $2006^{1}$. Cette forte prépondérance remonte aux débuts du protectorat français. Elle est liée à la situation de dépendance et à la présence du port et des services urbains (Kaioua, 1996). Toutes les tentatives

1 Enquête annuelle du ministère en charge de I'Industrie. 
de rééquilibrage menées depuis 1980 ont échoué, jusqu'au lancement du grand projet tangérois (2002). Celui-ci, qui valorise la situation avantageuse de la ville avec la construction d'un port majeur pour porte-conteneurs (Piermay, 2009), a stimulé une forte croissance de l'industrie. La part de l'emploi industriel de la Région de Tanger-Tétouan est ainsi passée de 9,6\% (1990) à 15,7 \% (2006) de l'emploi national, faisant pour la première fois reculer nettement celle de Casablanca, qui connaît aussi un certain desserrement vers la Chaouia voisine.

Une réelle réflexion spatiale a été développée dans le cadre de la politique industrielle marocaine. La création de deux pôles d'industries lourdes dans la sphère casablancaise, à Mohammedia (années 1960), puis à Jorf Lasfar à partir des années 1990, a desserré la nébuleuse centrale. Si des zones industrielles ont été aménagées à travers tout le pays, de manière parfois illusoire, les grandes orientations prises depuis l'avènement de Mohammed VI (1999) privilégient la notion de pôles de compétitivité, avec deux modes opératoires majeurs. Le premier définit de grands projets, investissements massifs en des espaces ciblés : Tanger (promue à un avenir de porte d'Europe), Rabat (équipement et embellissement de la ville capitale) et depuis peu Casablanca (modernisation de la métropole). Le second mode définit une stratégie industrielle. Établie par le cabinet international Mc Kinsey (programme Émergence-I et II, 2005 et 2009), celle-ci a identifié 8 secteurs d'activité susceptibles de constituer les «métiers mondiaux » du Maroc, et leurs implantations : avant tout, Casablanca (offshoring, électronique, aéronautique), Tanger (automobile, électronique), Rabat (offshoring), Agadir (agro-alimentaire, produits de la mer) et MeknèsFès (agro-alimentaire). Le programme Émergence-II s'attache à trois questions épineuses, le foncier, la formation, le financement. Sa décision-phare porte sur la création de vingt-deux parcs industriels intégrés (P2I) et spécialisés. Le ciblage des activités et l'établissement de priorités montrent que le pouvoir mise sur l'attractivité différenciée des espaces.

Contrastant avec cette prise en compte grandissante de l'espace, la politique industrielle marocaine se pose peu en termes de territoires. Le ministère en charge de l'aménagement avait lancé une étude sur les SPL (Courlet, 2006). Menée par un ministère de faible poids, elle n'eut pas de suite. L'injonction faite aux conseils régionaux de "décliner» le Programme Émergence-I montra surtout le poids des autorités déconcentrées, sauf à Agadir. S’il y eut des projets de clusters, encore mal définis et toujours fortement pilotés par le haut, les initiatives tendant à faire émerger du territoire sont modestes et peu signifiantes.

Cette faiblesse s'explique par le poids du pouvoir central, voire du roi, dans les processus de décision. Les grands projets sont royaux, pilotés par les conseillers du roi et des agences localisées à Rabat, même pas par les walis (préfets), pourtant nommés par le roi. Le gouvernement n'a qu'un rôle limité. Grâce à son entregent, Driss Jettou (2002-2006), un technocrate issu du patronat textile, avait acquis une certaine autonomie dans le domaine économique. Ce n'est pas le cas de son successeur, Abbas El Fassi, un notable du vieux parti conservateur de l'Istiqlal. Une constante est la faible mobilisation des acteurs locaux. Malgré 


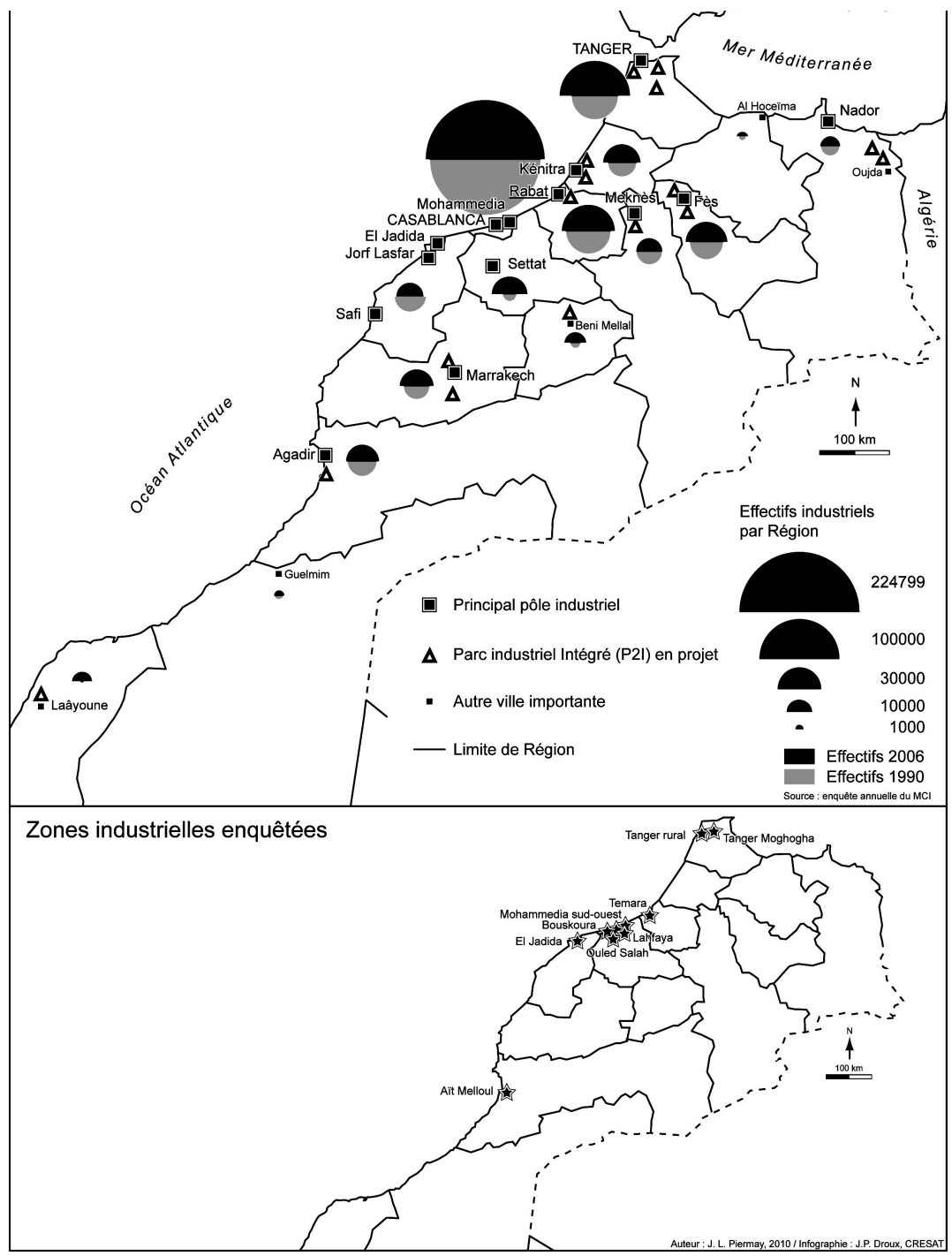

Fig. 1 Les espaces de l'industrie au Maroc

déclarations et textes, la décentralisation progresse peu. La presque totalité des conseils régionaux sont évanescents. Les conseils communaux sont en général des mosaïques d'intérêts particuliers, surtout fonciers; l'État les considère comme des obstacles à la modernisation et les neutralise dans ses grands projets par des dispositions dérogatoires. L' "autorité locale » est le wali, non pas l'élu. Et quand l'autorité locale a le souci de "faire territoire ", ce qui n'est pas sa préoccupation première dans une carrière hachée de postes successifs et soumise aux aléas du 
haut, ses relations personnelles lui font rechercher des alliés modernistes, comme des chefs d'entreprises bien introduits (Piermay et al., 2009).

\section{La territorialisation, un processus aux multiples facettes marqué par l'incomplétude}

Le caractère non fonctionnel du territoire dans les politiques publiques ne signifie pourtant pas l'inexistence d'un processus de territorialisation. En effet, les acteurs territorialisant ne se limitent pas aux pouvoirs publics. Mais du fait du poids de pouvoirs d'État peu enclins à territorialiser, il n'est qu'un processus émietté et multiple.

Tab. 1 Principales caractéristiques des espaces d'accueil enquêtés

\begin{tabular}{|c|c|c|c|c|c|c|c|}
\hline & \multirow{2}{*}{$\begin{array}{c}\text { Espace } \\
\text { d'accueil }\end{array}$} & \multirow{2}{*}{$\begin{array}{l}\text { Structure } \\
\text { de gestion }\end{array}$} & \multirow{2}{*}{$\begin{array}{c}\text { Distance } \\
\text { centre-ville } \\
\text { km }\end{array}$} & \multirow{2}{*}{ Sup. ha } & \multicolumn{2}{|c|}{$\mathrm{Nb}$ entreprises } & \multirow{2}{*}{$\begin{array}{l}\text { Nb emplois } \\
\text { moyen par } \\
\text { entreprise } \\
\text { enquêtée }\end{array}$} \\
\hline & & & & & Total & Enquêtées & \\
\hline $\begin{array}{l}\text { Agadir Aït } \\
\text { Melloul }\end{array}$ & ZI & A & 19 & 354 & 128 & 25 & 129 \\
\hline Casa Bouskoura & $\mathrm{PI}$ & $S$ & 19 & 28 & 43 & 32 & 36 \\
\hline Casa Lahfaya & QI & - & 10 & - & 28 & 21 & 52 \\
\hline $\begin{array}{l}\text { Casa Ouled } \\
\text { Salah }\end{array}$ & $\mathrm{ZI}$ & $\begin{array}{l}\text { A non } \\
\text { active }\end{array}$ & 20 & 105 & ? & 22 & 95 \\
\hline $\begin{array}{l}\text { Mohammedia } \\
\text { SW }\end{array}$ & ZI & A & $\begin{array}{l}23 \text { (Casa) } \\
4 \text { (M/dia) }\end{array}$ & 58 & 85 & 28 & 64 \\
\hline $\begin{array}{l}\text { Rabat Temara } \\
\text { Ain Attiq }\end{array}$ & $\mathrm{QI}+\mathrm{ZI}$ & - & 9 à 13 & - & 42 & 34 & 74 \\
\hline Tanger rural & diffus & - & 5 à 11 & - & $?$ & 13 & 60 \\
\hline $\begin{array}{l}\text { Tanger } \\
\text { Moghogha }\end{array}$ & $\mathrm{ZI}$ & A & 5 & 138 & 122 & 37 & 235 \\
\hline
\end{tabular}

Structure de gestion : A (association d'industriels), S (société de gestion).

Le parti pris de recherche a été de faire des processus de territorialisation une approche de terrain. Des investigations personnelles ont été réalisées de manière similaire de 2007 à 2009, sous la forme d'enquêtes auprès de 213 chefs d'entreprises employant au total 21400 personnes, et d'entretiens auprès d'acteurs publics disposant d'une capacité d'initiative et d'analyse. Les enquêtes ont été menées dans des espaces d'accueil d'entreprises, terme générique recouvrant des réalités différentes de reconnaissance administrative et d'équipements ${ }^{2}$. Le choix des quatre agglomérations urbaines (Casablanca (et Mohammedia), Tanger, Rabat, Agadir) et des espaces d'accueil a été fait de manière raisonnée et n’est pas

2 La «zone industrielle » (ZI) est un lotissement aménagé, qui offre des terrains équipés. Le «parc industriel » $(\mathrm{PI})$ ajoute des services d'accompagnement et un gardiennage. Le « quartier industriel » (QI) est un espace non loti et non aménagé. "Tanger rural » est un secteur d'industrialisation diffuse. 
représentatif. En revanche, quand cela était possible, l'échantillonnage a été représentatif dans les espaces d'accueil. Les faits de territorialisation ont été recherchés à travers les diverses facettes de la vie des entreprises, elle-même considérée à travers la vision de l'enquêté, en général le patron, parfois un membre de la cellule dirigeante (souvent un de ses enfants appelé à reprendre la succession). Il s'agissait de comprendre pour l'entreprise les formes de proximité agissante et la manière dont elle vivait cette proximité. Croisée avec le regard des autres acteurs selon une approche contradictoire qui permet de relativiser et d'approfondir les dynamiques à l'œuvre, cette recherche des logiques de proximité ouvre à la compréhension des mécanismes de territorialisation auxquels participent les entreprises. Le traitement a été double, d'abord analytique en fonction des formes de proximité vécues par les entreprises, ensuite synthétique en fonction des formes de territorialisation à l'œuvre dans les lieux étudiés.

Les formes de proximité vécues par les entreprises ont été réparties en 7 domaines (tableau 2). Chaque entreprise exprimant une forme de proximité agissante dans l'un de ces domaines se traduisait par un point. Le nombre total de points était finalement rapporté au nombre d'entreprises enquêtées (colonne 8). Ainsi, le score global de la zone industrielle d'Agadir Aït Melloul signifie que chaque entreprise enquêtée a exprimé en moyenne 3,92 formes de proximité de toutes sortes.

Les différences sont fortes entre les espaces d'accueil et entre les villes. Agadir et Mohammedia se détachent nettement en tête du classement. On peut interpréter le bas du classement de plusieurs manières : tous les espaces d'accueil de Casablanca s'y trouvent localisés ; les espaces non aménagés (Lahfaya, Tanger rural, voire Temara) également.

Les scores globaux ont des composantes très variées :

- la zone industrielle d'Agadir Ait Melloul, la plus originale avec des industries liées à l'agriculture, est un espace animé de rencontres entre industriels, agriculteurs et commerçants (col. 2), véritable point nodal de la petite région agricole du Souss (col. 7), dont la majorité des acteurs se reconnaissent une parenté culturelle en tant que "Soussis", des Berbères au dynamisme entrepreneurial connu. Cela facilite la dynamique associative (3). Des liens étroits existent entre les entreprises autour de la question des emballages (1), comme dans plusieurs sites d'enquêtes;

- la zone industrielle sud-ouest de Mohammedia se distingue surtout par les liens que les entreprises ont tissés avec les industries lourdes (raffinerie de pétrole, pétrochimie) du pôle voisin (col. 1). Plusieurs patrons ont commencé leur carrière comme cadres dans ces grandes entreprises et conservent avec elles de fortes relations commerciales. Les scores élevés de la colonne 7 sont plus liés à la proximité relative de Casablanca qu'à celle de Mohammedia ;

- la zone industrielle de Tanger Moghogha est connue par l'efficacité de son association d'industriels (3), en fait développée grâce à un holding associant des capitaux locaux et français (5). Les relations de production (1) sont moins significatives, souvent liées à de la sous-traitance. Il y a un attachement des 


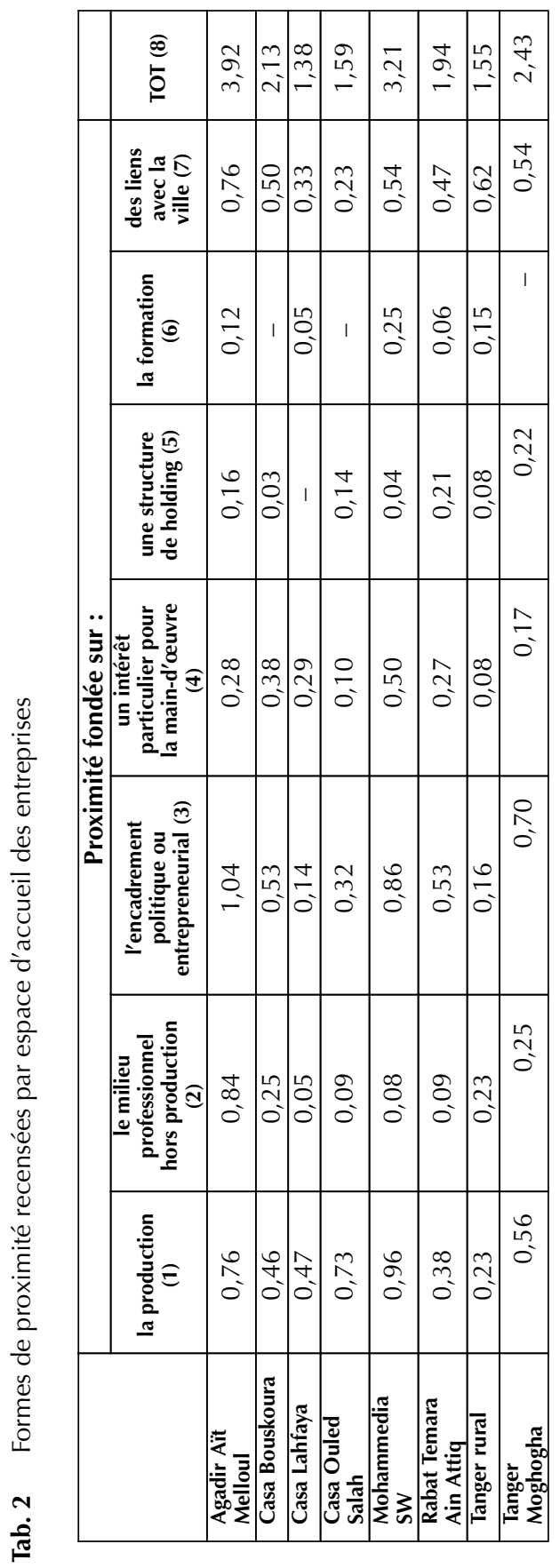


entrepreneurs à la ville, complété par des liens avec les montagnes du Rif et avec l'Espagne toute proche (7);

- les espaces d'accueil de l'ouest de Rabat ont plus qu'ailleurs des liens avec les autorités centrales (3). Un président de Conseil communal, ancien industriel et propriétaire de terrains dans les lieux, participe aussi au score de la colonne 3. La présence de holdings à base familiale, très attachés à Rabat, explique les résultats des colonnes 5 et 7 ;

- Casablanca a partout des scores très faibles, que l'aménagement soit bon (Bouskoura) ou catastrophique (Lahfaya), que la gestion soit bonne (Bouskoura) ou absente (Lahfaya, Ouled Salah). Pour des entreprises majoritairement de petite taille et situées en bout de chaîne de production, les relations productives sont faibles (1). Mais les « liens avec la ville» (7) ont été sous-estimés par les enquêtés, tellement la symbiose est évidente entre le tissu productif et la métropole.

Dans ce kaléidoscope, rares sont les points communs entre les espaces d'accueil. Mais on peut remarquer que les relations extérieures liées à la formation sont rares, que les relations liées à la $\mathrm{R} \& \mathrm{D}$ sont nulles et que les différences sont peu signifiantes sur les questions de personnel.

La question de la territorialisation fait apparaitre des lignes plus nettes. Les collectivités locales ne jouent un rôle (modeste) que lorsque leur responsable est personnellement intéressé, comme détenteur de terres (Ain Attiq, Lahfaya) ou industriel (Ain Attiq, Ait Melloul). Il est rare au Maroc qu'une vraie zone industrielle soit le fait d'une municipalité (mais il y en a une à Ain Attiq). Plus curieusement, les autorités déconcentrées apparaissent peu dans les enquêtes. Elles sont mentionnées à Mohammedia, mais derrière l'action de l'autorité locale se profile l'intérêt que le roi a manifesté pour un site qu'il avait visité en 2000. Un vrai partenariat avec l'autorité locale n'est signalé que pour l'association de la zone de Moghogha. Les acteurs administratifs et politiques ont peu de poids dans ces processus.

En fait, les processus de territorialisation à l'œuvre autour de la question productive dépendent essentiellement des entreprises elles-mêmes, et engagent la question de la confiance. La famille est le lien majeur qui donne la cohésion au capital, tant pour les petites sociétés que pour certains des plus gros holdings; elle ancre localement les entreprises, les arrime aux villes où elles sont installées (non nécessairement aux lieux d'origine). Le besoin de confiance explique aussi le rêve de beaucoup d'enquêtés, de développer " des relations familiales » avec le personnel, même si cela recouvre des types de rapports sociaux très différents, allant du clientélisme à des relations conviviales plus modernes. La question de la confiance est exprimée à son échelle la plus ample à Agadir, sous la forme d'une communauté culturelle réunissant des acteurs diversifiés. À Rabat Ain Attiq, la forme la plus évidente de proximité agissante est le holding, dans la mesure où des réserves foncières constituées de longue date hors zones dédiées permettent aujourd'hui de regrouper des entreprises, autour d'échanges en partie productifs et de services communs. Cette recherche de la confiance à travers des relations socialement éprouvées apparaît en contradiction avec le souci officiel 
de fonder l'essor industriel sur la captation d'investissements extérieurs (Piermay, Piveteau, 2009), facteur par ailleurs peu favorable à la construction de territoires. C'est pourtant sans compter avec des processus d'hybridation qui jouent sur le long terme. Ainsi, à Tanger Moghogha, la forme la plus évidente de proximité agissante est l'association locale d'investisseurs, à l'origine de laquelle se trouvent de grandes entreprises issues de joint-pentures des années 1980. Aujourd'hui, ce sont toujours elles qui apportent leur dynamisme à l'association, sous le regard indifférent ou envieux selon les cas de sociétés familiales souvent ancrées dans des logiques locales largement extra-industrielles.

Même si ces processus de territorialisation se sont développés autour de la question productive, c'est rarement la production elle-même qui les génère. Il y a pourtant de nombreux exemples de collaborations autour de la production. Mais ces liens sont dans l'ensemble peu ambitieux, du fait notamment de la faiblesse des industries de base.

À ce stade de l'analyse, on peut dire que la territorialisation existe à l'état de bribes autour de la question productive au Maroc. Les facettes sont diversifiées : plutôt «institutionnelle » à Agadir, "organisationnelle » à Tanger et Rabat, "géographique » à Casablanca (Pecqueur et Zimmermann, 2004), ordre qui exprime selon ces auteurs une intensité décroissante. Pourtant, l'impression prévaut d'un déficit général d'implication des pouvoirs publics, dont les structures poussent à une territorialisation d'échelle nationale. L. Jaïdi (2009) souligne ce déficit et appelle à « des dynamiques territoriales concertées ", pour lui élément nécessaire de relance de la politique industrielle. Mais au-delà de l'incomplétude actuelle, quelles sont les potentialités de la territorialisation au Maroc ? Quel peut être le sens d'une politique de territorialisation, et que peut-on en attendre ?

\section{Promesses et illusions du territoire}

Ces questions appellent à réexaminer la manière dont interfèrent le productif et le territoire, à travers une relecture aux contours plus larges.

\subsection{La territorialisation dépasse la question productive}

Les dynamiques sociales observées laissent souvent à l'activité industrielle un rôle secondaire. À Agadir, là où le processus est le plus abouti, les articulations se nouent autour des commerçants, les plus au contact des agriculteurs. C'est chez eux, vendeurs d'intrants et concessionnaires de machines agricoles, que le thé se boit en commun, que les réseaux sociaux se forment, fondant une sorte de SIAL (système agroalimentaire localisé) (Muchnik, 2002), qui entraîne les industries de conditionnement dans la dynamique locale.

La question productive peut occuper une place encore plus subalterne. Ainsi, beaucoup de chefs d'entreprises familiales de Tanger jouent sur plusieurs tableaux (industrie, spéculation foncière et immobilière, fonctions électives locales et trafics). Souvent originaires du Rif, dotés d'une importante clientèle, ils ont des 
comportements très opportunistes. Dans un contexte urbain marqué par le grand projet royal, ils privilégient la spéculation et abandonnent l'usine à leurs enfants tout en gardant la haute main sur le groupe familial.

De plus, la territorialisation n'est pas un processus simple et porteur de toutes les vertus (Fauré et al., in Antheaume et al., 2005). Majoritairement installées à Rabat Témara, les entreprises de transformation du liège ont proposé avant la lettre un projet de parc industriel intégré. Mais ces liens s'inscrivent dans une situation économique difficile (concurrence du bouchon en plastique) et urbaine (les vastes espaces de stockage sont convoités). Cette territorialisation désespérée diffère de celle d'Agadir, plus prometteuse.

\subsection{Un processus en cache-t-il un autre?}

Les autorités marocaines semblent actuellement plus enclines qu'auparavant à favoriser une territorialisation vertueuse. Ainsi, le P2I concrétise apparemment une rencontre entre les autorités et les entreprises, "intégration » signifiant recherche de synergie et mobilisation des pouvoirs publics autour d'une filière industrielle.

Le problème est que la zone industrielle n'a pas été jusque-là un facteur de proximité agissante. Les scores plus élevés attribués dans l'enquête aux espaces aménagés cachent des biais : à Casablanca où l'offre est insuffisante, ils apportent surtout un surcroît de respectabilité. Les associations d'entrepreneurs n'existent pas partout et ne fonctionnent pas toujours. Même quand ils existent, les services communs ne créent pas nécessairement de liens entre les entreprises (Bouskoura). Les procédures de sélection des entreprises ne privilégient jamais les complémentarités fonctionnelles. Au mieux, on juge les chances de réussite individuelle des projets, et souvent le poids des réseaux sociaux ou la perspective de rentabilisation commerciale de l'aménagement emporte la décision.

Les P2I changeront-ils la donne ? Pour les entreprises, il s'agit d'abord d'une réponse à la délicate question du foncier, principal obstacle à l'investissement au Maroc (Piermay, 2010). Pour les autorités centrales, qui ont d'abord puisé dans le domaine public de l'État, le problème est de trouver des espaces, en surmontant l'inertie des intérêts fonciers privés, qui tiennent les collectivités locales. Le discours de la territorialisation risque de se limiter à un essai de résolution de l'épineuse question foncière.

\subsection{Le primat de la métropole}

Les enquêtes montrent de très fortes tonalités urbaines, les spécificités économiques cachant souvent des originalités ancrées dans les sociétés. Celles-ci renvoient aux identités chez les Soussis d'Agadir; à une hybridation entre le milieu local et l'extérieur à Tanger, peut-être héritage du passé international, sinon interlope, de la ville.

L'analyse est plus difficile à Casablanca, où les proximités agissantes apparaissent faibles. Pourtant, de nombreux chefs d'entreprises, très attachés à la ville, moins parce que c'est la leur que parce que c'est le lieu où il faut être, 
semblent contredire cette idée. Il est étonnant d'entendre des patrons installés dans des conditions catastrophiques (absence de statuts fonciers officiels, piste d'accès défoncé, non-raccordement au réseau d'eau) évoquer leur satisfaction quant à leur localisation. Tout converge vers l'existence à Casablanca d'avantages tellement évidents qu'on ne les évoque même pas. Des avantages internes à la grande ville : la variété des services, le port en premier, un marché diversifié de la main-d'œuvre, les relations sociales, la richesse d'une vie urbaine à défaut d'une réelle qualité de vie. Des avantages liés à la situation de hub du Maroc : les infrastructures, l'accès aux fournisseurs et au marché. Par ailleurs, la conception de la grande ville est extensive. Rabat apparait comme une localisation par défaut, la localisation massive des décideurs politiques pesant d'un poids extrêmement réduit. En ce qui concerne "les liens à la ville », les enquêtés pensent Casablanca et métropole, non pas Mohammedia, et parfois non pas Rabat. Ainsi, à l'écart des embouteillages de Casablanca, toutes les zones industrielles situées entre Casablanca et Rabat sont indistinctement vues comme " la meilleure localisation du pays ». Malgré le grand projet royal, la situation de Tanger, encore très peu apte à répondre aux besoins des entreprises (surtout en matière de fournisseurs et de clients), n'est pas encore acquise. Même celle d'Agadir est précaire : les exploitations agricoles sont menacées par le manque d'eau, des entrepreneurs souhaitent partir et le dynamisme soussi pourrait se reconstituer à Casablanca, où il n'est pas absent. Tous ces faits donnent à penser que la proximité n'est nulle part aussi forte et aussi agissante qu'à Casablanca. L'expression "proximité géographique » telle qu'utilisée par Pecqueur et Zimmermann (2004) a un sens trop faible. Au Maroc en tout cas, la proximité «métropolitaine » est primordiale.

\section{Conclusion}

Le titre de la communication posait d'une manière provocante l'hypothèse d'une attractivité qui se ferait sans recours au territoire. Par le poids de l'échelon central et par l'incomplétude des systèmes d'acteurs locaux, le Maroc permettait de poser cette hypothèse. C'était sans compter avec la métropole. Forme actuelle la plus accomplie de la ville, celle-ci offre le maximum de possibles (Claval, 1981). Hub qui résout le mieux le paradoxe entre le global et le local, la métropole aide à faire face à l'incertitude (Cavallier, 2005). La métropole est aussi potentiellement le meilleur intégrateur des proximités, sous réserve toutefois qu'une recherche volontariste des cohérences l'emporte sur les blocages. De fait, avec le début d'un grand projet global et celui d'une concertation autour de l'aménagement de la Région, Casablanca se trouve désormais et de nouveau au cœur des enjeux du pays.

Si le Maroc offre peu d'exemples de création de territoire par le productif, ce fut pourtant le projet sur le long terme de Casablanca, celui qui produisit la métropole d'aujourd'hui. C'est aussi en quelque sorte l'enjeu du grand projet de Tanger. Mais Tanger n'est pas Mohammedia, tellement proche de Casablanca 
que la réussite de ce pôle d'industries de base ne pouvait être que synergique avec le desserrement de la grande ville. À Tanger, la maturation métropolitaine sera plus difficile à réaliser qu'un port.

Au Maroc, la suppléance des acteurs locaux par des autorités déconcentrées ne favorise pas la construction d'articulations complexes et de territoires. Ces situations proconsulaires facilitent le lancement des projets, et en limitent finalement l'efficacité. On observe plutôt des bribes de territoires, qui se greffent tant bien que mal sur des dynamiques insufflées d'en haut ou qui se forment en marge des pouvoirs centraux. Sur le plan des politiques publiques, le territoire risque de n'être qu'un thème de discours à la mode, qui ne résistera pas à la sanction du long terme.

Plus qu'une coalition d'acteurs, le territoire est en effet un processus complexe, résultant d'une construction et d'une déstructuration incessantes (Raffestin, 1986), d'une grande richesse articulatrice (Lévy et al., 2003 ; Pecqueur et al., 2004) : entre matérialité et symbole, espace et réseau, contrôle et sentiment d'appartenance. Dans un environnement mondialisé, il est un véritable paradoxe, celui de l'autonomie du local dans l'interdépendance (Barel, 1981). C'est ce qui se réalise dans la métropole, qui offre une proximité complexe, longuement mûrie. La proximité vertueuse se limiterait-elle au Maroc à la métropole ? Pour l'essentiel, on peut le penser, mais deux pistes ouvrent des possibilités. La première est qu'une proximité agissante dans le champ de la production est susceptible de constituer un «plus », même dans une métropole. La seconde est que la métropole tend aujourd'hui à se diffuser, à la fois à la faveur de l'amélioration des infrastructures, de la saturation casablancaise et d'une dynamique contagieuse. La métropole est donc un réel gisement encore à exploiter.

UFR de géographie
Université de Strasbourg
3, rue de l'Argonne
67083 Strasbourg Cedex
jlp@unistra.fr

\section{Bibliographie}

Antheaume B., Giraut F. (2005). Le Territoire est mort, vive le territoire !, Paris, IRD Éditions, 384 p.

Barel Y. (1981). "Communication paradoxale et reproduction sociale », Colloque sur la théorie de la communication. Universidad Internacional Menendez Pelayo, Santander, 33 p.

Burger M.-J., Van Oort F.-G., Frenken K., Van der Knaap B. (2009). « Networks and Economic Agglomerations: Introduction to a special issue », Tijdschrift voor Economische en Sociale Geografie, vol. 100, $\mathrm{n}^{\circ} 2$, p. 139-144.

Cavallier G. (2005). « La ville dans l'économie globale », in Lollier J.-Ch., Prigent L., Thouement H., Les Nouveaux Facteurs d'attractivité dans le jeu de la mondialisation. Rennes, Presses Universitaires, 235 p. (p. 19-37).

Claval P. (1981). La Logique des villes. Essai d'urbanologie, Paris, LITEC, 634 p. 
Courlet C. (dir.) (2006). Territoire et développement économique au Maroc. Le cas des systèmes productifs locaux. Économie critique, Rabat, Paris, L'Harmattan, $161 \mathrm{p}$.

Jaïdi L. (2009). «Émergence II : le triangle des 3F », La Vie ECo, 27 février 2009, p. 28.

Kaioua A. (1996). Casablanca, I'industrie et la ville. URBAMA, Communauté Urbaine de Casablanca, fascicule de recherche $\mathrm{n}^{\circ} 30,2$ tomes, $775 \mathrm{p}$.

Levy J., Lussault M. (2003). Dictionnaire de la géographie et de l'espace des sociétés, Paris, Belin, $1024 \mathrm{p}$.

Muchnik J., Sautier D. (1998). Séminaire "Systèmes agro-alimentaires localisés et construction de territoires ", Montpellier, CIRAD.

Pecqueur B., Zimmermann J.-B. (2004). Économie de proximités, Paris, Lavoisier, Hermès, 264 p.

Piermay J.-L. (2009). « La frontière, un outil de projection au monde. Les mutations de Tanger (Maroc) », Espace et société, p. 69-83.

Piermay J.-L., Piveteau A. (2009). «L'impensé local de la stratégie d'émergence industrielle marocaine. Quelques limitations observables à partir du cas tangérois », in Mezouaghi M., Les Localisations industrielles au Maghreb ; attractivité, agglomération et territoires, Tunis-Paris, IRMC-Karthala, p. 209-232.

Piermay J.-L. (2010). « Le développement durable au péril de la question foncière », communication au colloque Les Villes durables au Maghreb et en Méditerranée : enjeux scientifiques et enjeux opérationnels. IRMC, CEDEJ, Caisse des Dépôts et Consignations, AFD, AUF, Hammamet.

Rallet A., Torre A. (2007). Quelles proximités pour innover?, Paris, L'Harmattan, 221 p.

Raffestin C. (1986). "Écogenèse territoriale et territorialité », in Auriac F., Brunet R., Espaces, jeux et enjeux, Paris, Fayard et Fondation Diderot, Nouvelle Encyclopédie des Sciences et des Techniques, p. $173-186$. 